\title{
A method using biodegradable polylactides/polyethylene glycol for drug release with reduced initial burst
}

\author{
Yi-You Huang ${ }^{\mathrm{a}, *}$, Tze-Wen Chung ${ }^{\mathrm{b}}$, Tzeng-Wen Tzeng ${ }^{\mathrm{b}}$ \\ a Institute of Biomedical Engineering, College of Engineering, National Taiwan University, Taipei, Taiwan, ROC \\ ${ }^{\mathrm{b}}$ Department of Biomedical Engineering, Chung-Yuan University, Chung-Li, Taiwan, ROC
}

Received 19 October 1998; received in revised form 8 February 1999; accepted 9 February 1999

\begin{abstract}
Post-coating of biodegradable polylactides (PLA)/polyethylene glycol (PEG) microspheres with a gelatin film produced by dipping the microspheres into a gelatin solution to reduce the initial drug release burst was investigated. Biodegradable block PLA/PEG microspheres, prepared by $\mathrm{o} / \mathrm{w}$ emulsion/solvent evaporation, showed that the hydrophilic segment PEG protruded to the sphere surface. However, these microspheres also showed a large burst release in the initial period. Post-coating of the PLA/PEG microspheres with a gelatin film by dipping the microspheres into a dilute gelatin solution effectively inhibited the initial burst release rate in the drug release tests. Post-coating of gelatin reduced $98 \%$ of the burst release. With thicker coatings, there were slower releasing rates, and the release rate can be simply related to the coating thickness. (C) 1999 Elsevier Science B.V. All rights reserved.
\end{abstract}

Keywords: Biodegradable polymers; Drug release; PLA/PEG; Microencapsulation

\section{Introduction}

Biodegradable polylactides (PLA) and its copolymers have attracted much attention as materi-

\footnotetext{
* Corresponding author. Present address: Institute of Biomedical Engineering, College of Medicine, National Taiwan University, No.1, Sec. 1, Jen-ai Road, Taipei, Taiwan, ROC. Fax: + 886-2-23940049.

E-mail address: yyhuang@ha.mc.ntu.edu.tw (Y.-Y. Huang)
}

als for drug delivery. They are biodegradable, and are classified as minimally toxic and shown not to cause adverse tissue reaction (Gogolewski et al., 1993; Gupta et al., 1997). There is no need to retrieve or retreat the carrier after drug depletion. Sampath et al. (1992) fabricated poly(L-lactic acid) implants containing gentamicin sulfate for the treatment of osteomyelitis by prolonged localized delivery of drug. In their in-vitro release experiments, they showed that the PLA implants 
have an improved extent of release in comparison with the conventional nonbiodegradable PMMA implants that were incomplete and poorly controlled drug release during the same time period. However, in a small cavity, such as in bone intramedullary lumen, poly(L-lactic acid) degrades into lactic acids. It creates a local acidic environment that may be harmful to the surrounding tissue. The $\mathrm{pH}$ of a suspension of PLA in saline at a concentration of $1 \% \mathrm{w} / \mathrm{w}$ is as high as 2.8 (Miyamoto et al., 1993). Meanwhile, high molecular weights of poly(DL-lactic acid) homopolymers were inappropriate as a bone morphogenetic protein (BMP) carrier, primarily because they produced strong foreign-body reactions or chronic inflammation and were too slowly absorbed to be replaced by induced bone (Miyamoto et al., 1992). Introducing polyethylene glycol into polylactic acid homopolymers could increase the hydrophilicity of the polymer carriers, increase the degradation rate and decrease the acidity of degraded products (Cohn and Younes, 1988; Zhu et al., 1990).

Modification of polymers by polyethylene glycol (PEG) has also been used to prolong the blood circulation of these polyester nanospheres. Gref et al. (1994) grafted PEG to biodegradable polyester, PLGA, by the method of using monomethoxy ethylene glycol reacting with lactide and glicolide. Core-corona type nanospheres were formed (Hrkach et al., 1997). These nanospheres showed long blood circulating properties. Conventional microspheres, such as albumin microspheres or polyester microspheres, will be rapidly captured by the reticuloendothelial system (RES) when administrated intravenously. Surface modification of collodial drug carriers by adsorption of block copolymers polyxamer reduced their phagocytic uptake by cultured phagacytic cells in vitro (Illum et al., 1987; Rudt et al., 1993). This modification also reduced uptake by the RES in vivo and increased blood residence time. Polyxamer is a mixture of polyethylene oxide (PEO, or PEG) and polypropylene oxide (PPO), and the presence of surface-orientated PEO on polystyrene microspheres will decrease the extent of protein adsorption (opsonisation) and phagocytosis of the microsphere by non-parenchymal liver cells.
In addition to being used as drug carriers, PLA/PEG copolymer could also be good candidates for scaffolds in tissue engineering for cell organ culturing. Patel et al., (1998) used PLAPEG-biotin copolymers to spatially control cell adhesion and control the directional growth of neurite extensions. However, PLA/PEG drug carriers showed a large burst effect (Huang et al., 1997). In this study we developed an effective method to produce PLA/PEG microspheres with reduced initial burst. This was accomplished by post-coating PEG/PLA microspheres with a gelatin film by dipping the microspheres into a gelatin solution. These microparticles can be used as vaccine carrier or be fabricated into implants, tablets, such as in the treatment as well as prophylaxis of bone infections.

\section{Materials and methods}

\subsection{Materials}

Propranolol hydrochloride (Nacalai Tesque, Kyoto, Japan) and lidocaine (Sigma Chemical, St. Louis, MO) were used as model drugs and used as received. PEG (molecular weight 2000 and 6000) and PVA (degree of polymerization $\cong 2000 ; n=$ 1500-1800) were also obtained from Nacalai Tesque. Methylene chloride and chloroform, LC grade, were obtained from Alps Chemical, Hsinchu, Taiwan. L-Lactide was purchased from DuPont, Wilmington, DE. All other regents were reagent HPLC grade, and used as received. Gelatin (average molecular weight $60000-$ $100000)$ was purchased from Wako Chemicals, Japan. Water was obtained from a purification system (Milli-Q, Millipore, Milford MA).

\subsection{Fabrication of biodegradable polylactides/PEG microspheres}

PLA/PEG copolymer was synthesized from Llactide dimer and PEG with number-average molecular weight 2000 . The polymerization was held at $180^{\circ} \mathrm{C}$ under a nitrogen atmosphere, and 0.05 wt. \% stannous octoate was added. Reaction time was kept $10 \mathrm{~h}$. Model drug, lidocaine, was 
encapsulated using an o/w emulsion technique. While for propranolol hydrochloride, double emulsion, w/o/w, was used in drug encapsulation. The aqueous drug solution $(1 \mathrm{ml})$ was first poured into the polymer solution (100 $\mathrm{mg}$ of polymer dissolved in $4 \mathrm{ml}$ methylene chloride or chloroform) to form a w/o emulsion. The w/o emulsion was then rapidly poured into $20 \mathrm{ml}$ of water containing $0.4 \mathrm{wt} . \%$ of poly(vinyl alcohol). The mixture was sonicated at an ultrasonic cleaner (Crest Ultrasonic, Model 275T, Treton, NJ) for $15 \mathrm{~min}$ to form an emulsion, then mechanically stirred with a magnetic stirrer (Corning, Stirrer/ Hot Plate, USA). Stirring was continued until the organic solvent had evaporated. The microspheres were then separated, and dried under vacuum.

Particle size of microspheres was determined by photon correlation spectroscope ( $\mathrm{He} / \mathrm{Ne}$ laser particle size analyzer, Malvern Instrument, Worcestershire, UK). ${ }^{1} \mathrm{H}-\mathrm{NMR}$ spectrum was measured with a Bruker AMX-400MHz spectrometer using solvent as the internal standard. The drug content was determined by dissolving a fixed amount of microparticulates in methylene chloride, and then measuring the drug content in an UV spectrophotometer (Beckman DU-64, Palo Alto, CA), at a wavelength of $214 \mathrm{~nm}$.

\subsection{Post-coating of biodegradable \\ polylactides/PEG microspheres with a gelatin}

Post-coating of PLA/PEG microspheres with gelatin was achieved by soaking the microspheres ( $0.5 \mathrm{~g}$, approximately) in either a 0.5 or $5 \%$ gelatin solution $(30 \mathrm{ml})$ for $5 \mathrm{~min}$ at room temperature (about $25^{\circ} \mathrm{C}$ ), then separating the microspheres from solution by filtration. The separated microspheres were dried in an oven at $40^{\circ} \mathrm{C}$ for $24 \mathrm{~h}$.

\subsection{Drug release test}

Microspheres, $0.1 \mathrm{~g}$, were poured into a $7 \mathrm{~mm}$ diameter die and compressed by $100 \mathrm{~kg} \mathrm{~cm}^{-2}$ hydro-pressure forming a tablet, $7 \mathrm{~mm}$ in diameter and $3 \mathrm{~mm}$ in thickness.

The release (dissolution) tests were carried out at $37^{\circ} \mathrm{C}$ in a shaken water bath. A test tablet $(0.1 \mathrm{~g})$ was placed into a tightly capped vial con- taining $5 \mathrm{ml} 0.025 \mathrm{M}$ phosphate buffer ( $\mathrm{pH} 7.4$ ) and brought to ionic strength 0.13 with sodium chloride. A sample $(200 \mu \mathrm{l})$ from the vials was withdrawn at preset intervals to determine the drug concentration. The same volume of fresh buffer was added each time that a sample taken. Drug concentration in the solution was assayed by capillary electrophoresis, with borate buffer, $\mathrm{pH}$ 9.4, as a running buffer. The sample mixture was separated by a high voltage, $15 \mathrm{kV}$, and measured by a UV detector at $214 \mathrm{~nm}$. The detection limits of drug concentration was $0.5 \mu \mathrm{g}$ $\mathrm{ml}^{-1}$.

\section{Results and discussion}

ABA block copolymers of polyethylene glycol and poly-L-lactic acid were prepared by ring opening polymerization of L-lactide with polyethylene glycol, $M_{n} 2000$ as previously described (Huang et al., 1997). The PEG weight percent in the feed stream was 3 or $9 \%$, respectively. The PEG weight percent in the resulting copolymer (i.e. $9 \%$ in the feed stream) was $14.1 \%$ as calculated from the ${ }^{1} \mathrm{H}-\mathrm{NMR}$ spectrum as shown in Fig. 1(a). The molecular weight $\left(M_{n}\right)$ of the resulting copolymer was 11702 . The particle size of microspheres made from the w/o/w emulsion solvent evaporation technique was $163.1 \pm 17$ $\mu \mathrm{m}$. The microspheres made from these polymers also showed core-corona characteristics when emulsion solvent evaporation technique was employed in the encapsulation process, as can be confirmed by ${ }^{1} \mathrm{H}-\mathrm{NMR}$ spectroscopy. Fig. 1 shows the ${ }^{1} \mathrm{H}-\mathrm{NMR}$ spectra of PLA/PEG (9\%) microspheres: (a) redissolved in $\mathrm{CDCl}_{3}$; and (b) as a suspension in $\mathrm{D}_{2} \mathrm{O}$. Core-corona microspheres occur mainly via the amphiliphic characteristics of PEG/PLA copolymers (Hrkach, et al., 1997). PEG has a good structural fit with $\mathrm{H}_{2} \mathrm{O}$, forming a gauche conformation suitable for hydrogen bonding between the water molecules and the ether oxygen of PEG in the ABA block copolymer. The high affinity of PEG with water will be pulled the PEG to the surface of micelles by the bulk water molecules in the emulsion process. At the same time, the hydrophobic polylactide seg- 
a

PLA/PEG COC13 112797

⿷

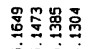

认ิ

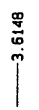

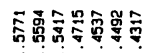

近

$\mathrm{CH}_{3}$
$\stackrel{\mathrm{CH}}{\mathrm{CH}}-$

$-\mathrm{CH}_{2}{ }^{*}-\mathrm{CH}_{2}{ }^{*}-\mathrm{O}-$

$\stackrel{\mathrm{CH}}{\mathrm{CH}}_{-}^{\mathrm{CH}^{*}}$

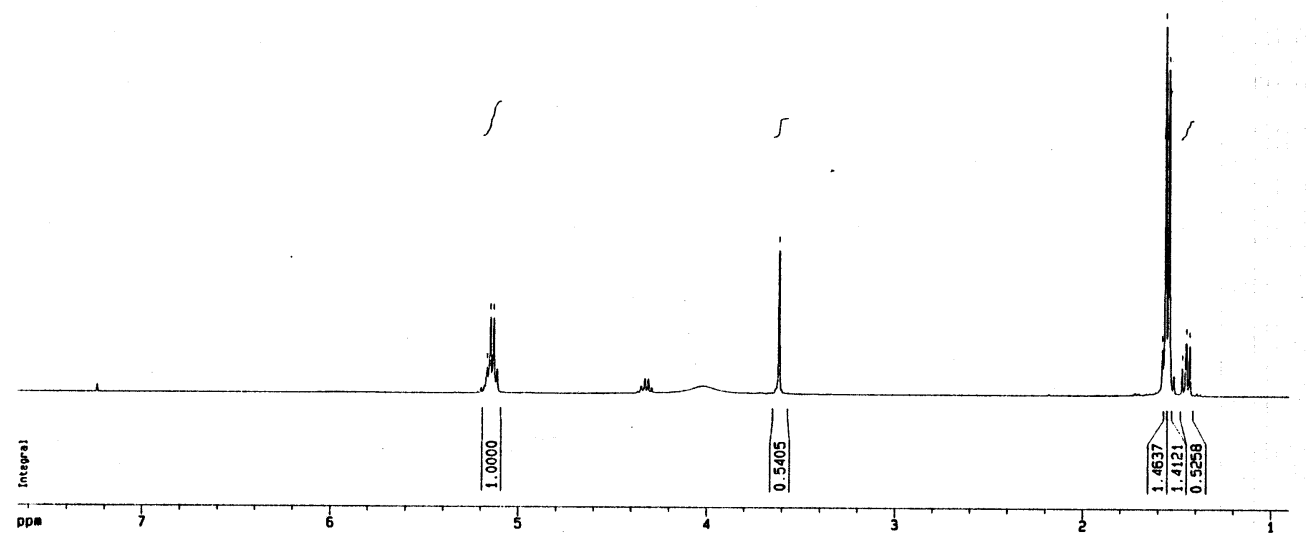

b

PLA/PEG D20 112797

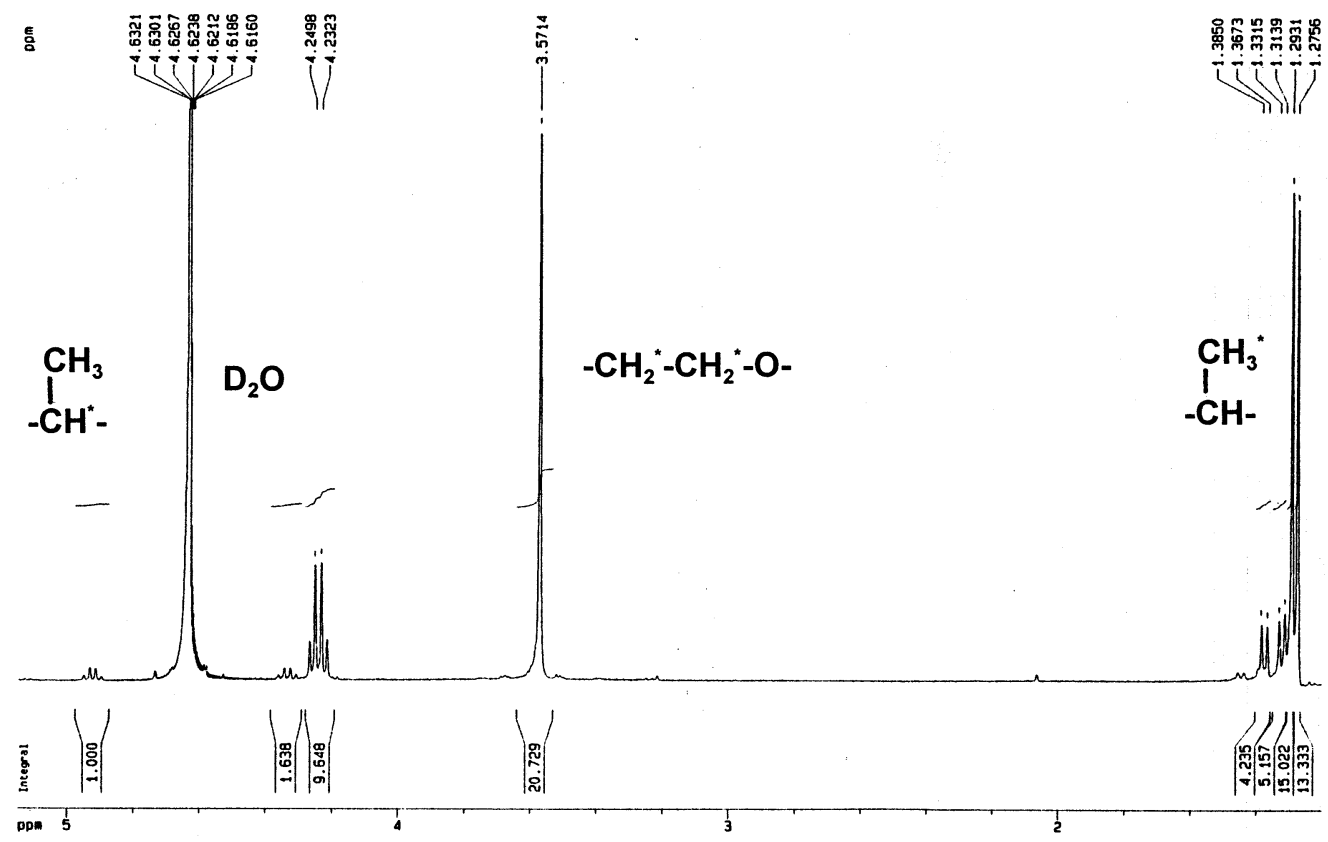

Fig. 1. ${ }^{1} \mathrm{H}-\mathrm{NMR}$ spectra of PLA/PEG $(9 \%)$ microspheres: (a) redissolved in $\mathrm{CDCl}_{3}$; and (b) as a suspension in $\mathrm{D}_{2} \mathrm{O}$. 
ments will be directed into the inner core of micelles during the emulsion process. After solidification during emulsion solvent evaporation, the PEG chains protrude into the dispersion medium, forming a hydrophilic particle surface during the emulsion solvent evaporation process, as shown in the NMR spectrum, Fig. 1. This type of drug carrier will has long blood residence time and can avoid RES uptake in vivo. The PEG outer layer in the corona-core microspheres increases the hydrophilicity of the surface, such that nonspecific interaction with liver Kupffer cells is reduced. Alternatively, PEG may sterically prevent the binding of serum opsonins to the particle surface, thereby reducing the affinity for RES.

Because of the high water solubility of PEG, the inclusion of hydrophilic PEG segments into the PLA polymers may also enhance the diffusivity of water or drug in the PLA/PEG copolymerdrug carriers (Zhu et al., 1990; Huang et al., 1997). PEG/PLA drug carriers show a large burst effect, as shown in Fig. 2. Increasing the PEG

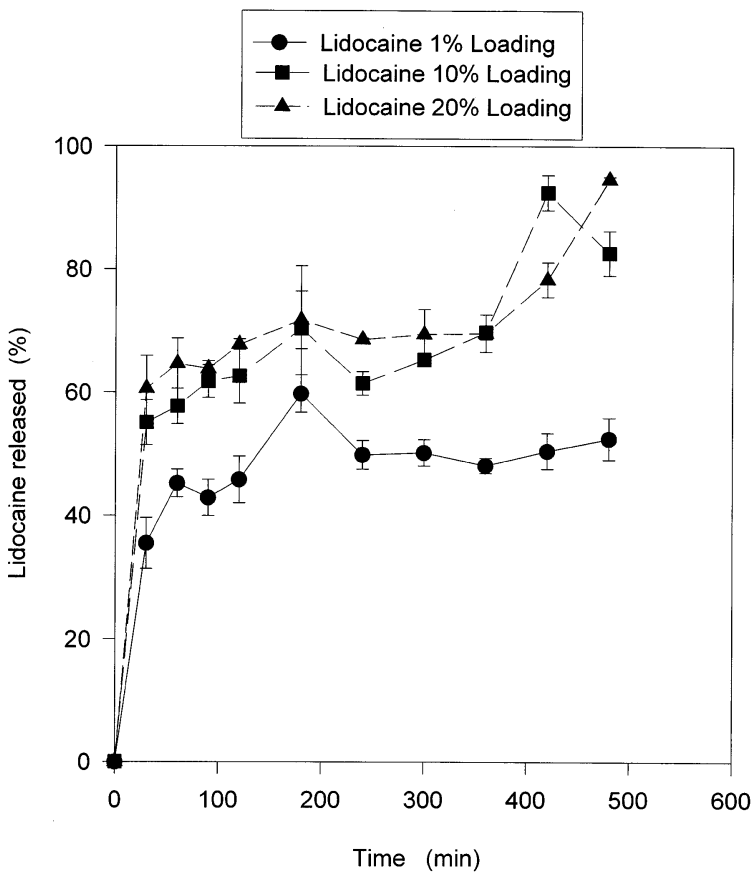

Fig. 2. Drug release from PLA/PEG (3 wt.\%) copolymers. Large burst release shown in the initial period. Data are the average of triplicate experiments, mean \pm SD.

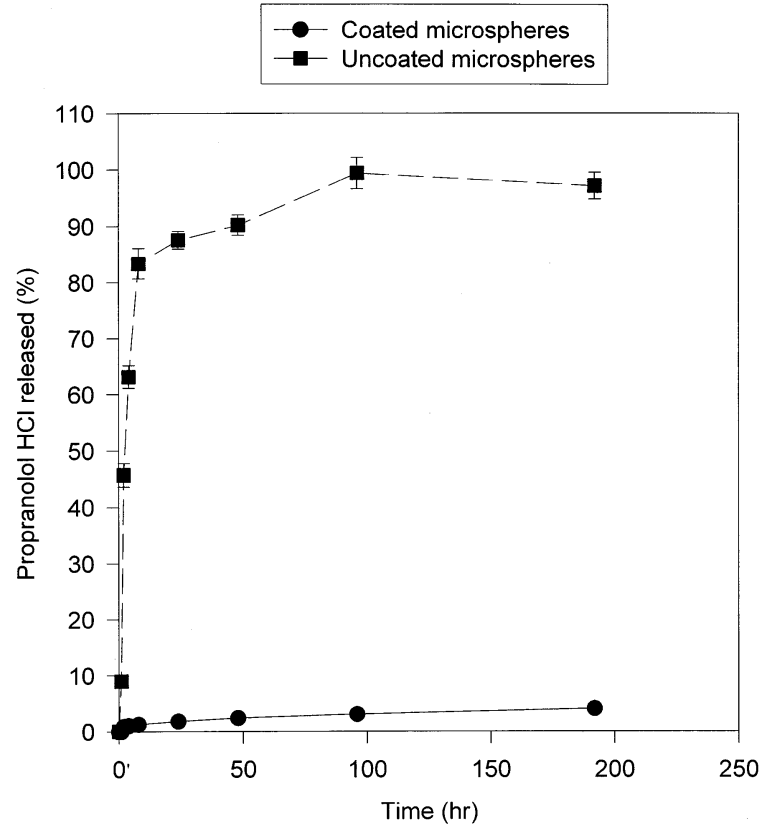

Fig. 3. Comparison of drug release from PLA/PEG (9 wt.\%) copolymers with and without gelatin post-coating. Data are the average of triplicate experiments, mean \pm SD.

contents will increase the drug diffusivity and decrease the molecular weight as well as $T_{\mathrm{g}}$ temperature.

Due to the higher hydration characteristic of PEG in the PLA/PEG copolymer, microspheres made from these copolymers had poor formation. Open cell channels were formed in the emulsionsolvent evaporation process because of the high interaction between PLA/PEG copolymer and nonsolvent water in the emulsion process. In addition, for most biodegradable polyester polymers, in the early stage of drug release, diffusional transport is the dominant release mechanism. The hydrophilic drug was easily dissolved and released through the porous channels, and a larger initial burst occurred.

In order to inhibit the initial drug burst, gelatin, which is a natural biodegradable macromolecule, was coated on the surface of microspheres. Fig. 3 shows the effect of post-coated gelatin on the drug release behavior of PLA/PEG $(9 \%)$ microspheres. Post-coating the microspheres with a gelatin film effectively inhibited the initial burst of 
the drug from the PLA/PEG microspheres. Postcoating of a gelatin film inhibited almost $98 \%$ of the initial burst.

Fig. 4 shows that post-coating a gelatin film not only reduced the initial burst, but also controlled the drug release rate. The release rate was far lower than from a simple gelatin film (Po and Mhando, 1984). It implied that there was probably hydrogen bond formed between PEG and gelatin molecules. The release rate of microspheres with drug loading $40 \%$ was higher than that of those with drug loading $20 \%$. In addition, higher concentrations of gelatin resulted in better control release of the drug (Fig. 5). Thus, the preprogrammed release rate can be manipulated by dipping the microspheres into different solutions of various gelatin concentrations.

The drug release rate of microspheres postcoated with gelatin was closely related to the permeation coefficient, $D K / \delta$, where $D$ is the drug diffusivity in the coating film, $K$ is the partition coefficient, and $\delta$ is the coating thickness. If the diffusivity $D$ and partition coefficient $K$ of drug in

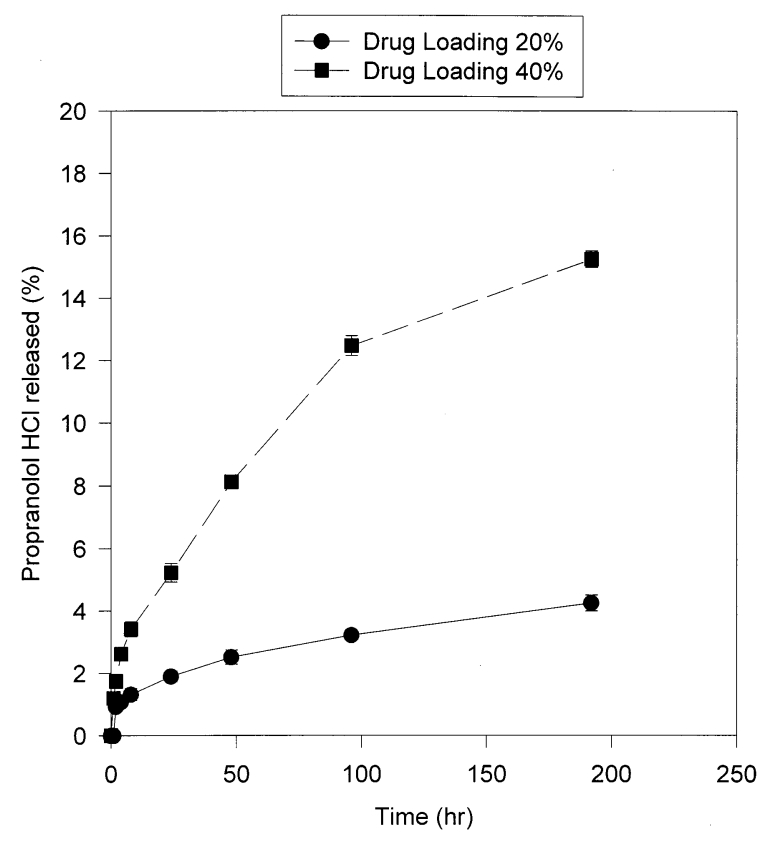

Fig. 4. Drug release from PLA/PEG (3 wt.\%) copolymers post-coated with gelatin for different drug loading. Data are the average of triplicate experiments, mean $\pm \mathrm{SD}$.

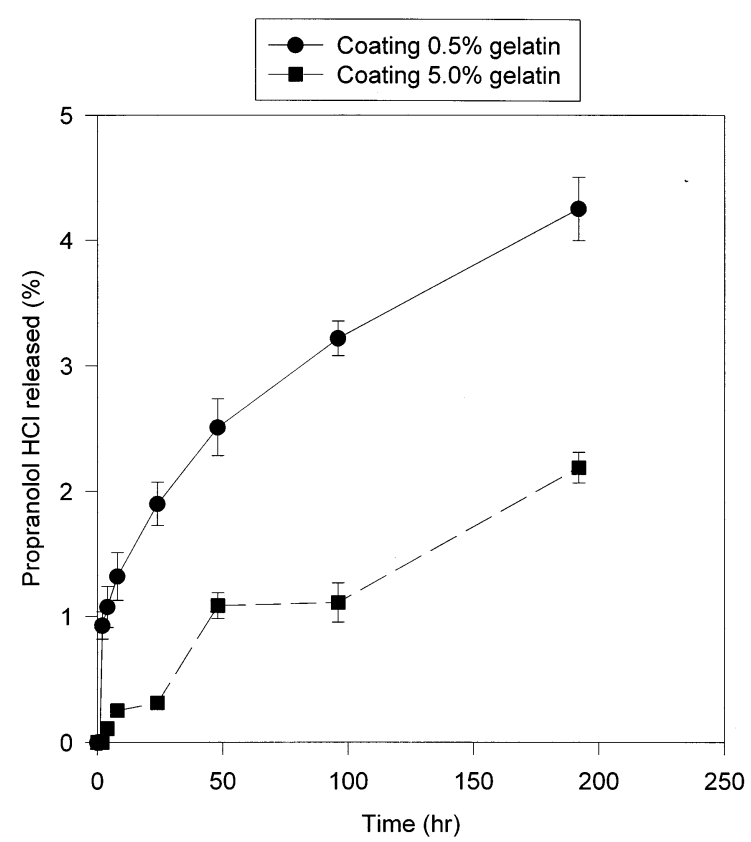

Fig. 5. Cumulative release amount of microparticulates made by 9 wt. $\%$ PEG/PLA copolymer post-coated with different gelatin concentrations. Data are the average of triplicate experiments, mean $\pm \mathrm{SD}$.

the coating film were kept constant, then the permeation coefficient would be inversely proportional to the coating thickness.

In the dipping process, the coating thickness could be approximated by the hydrodynamics of spherical falling films. The film thickness at any latitude is given as (Singh and Stockar, 1996),

$\delta=\delta_{1}(\operatorname{Sin} \alpha)^{-2 / 3}$

where $\delta_{1}$ is the film thickness at the equator $(\alpha=\pi / 2)$. This value is given by

$\delta_{1}=(3 Q \gamma / 2 \pi R g)$

where $Q$ is the volumetric flow rate of the film, $R$ is the radius of the sphere and $\gamma$ is the kinematic viscosity of the fluid. The influence of the polymer on the viscosity of the gelatin solution is closely related to the frictional effects encountered in sedimentation and diffusion (Flory, 1967). The viscosity of the solution as a whole is equal to the rate of energy dissipation divided by the square of the rate of shear. Hence, on the assumption that 
the solution is so dilute that there is no appreciable interaction between different polymer molecules, in either $0.5 \%$ or $5 \%$ gelatin solution, the contribution that the polymeric solute makes to the total viscosity is therefore

$\eta=\eta_{0}+\left(c N / M_{0}\right) \xi \bar{s}^{2} / 4$

where $c N / M_{0}$ is the number of polymer molecules per cubic centimeter, and $c$ is the concentration in grams per cc. In a very dilute solution, the variation of flow rate (viscosity) is not significant. Therefore, when dried, the film coating thickness on the PLA/PEG microspheres was proportional to the polymer concentration, i.e. $\delta \propto c$.

Based on a known permeation data, the theoretical predication of release rate for $5 \%$ gelatin post-coated microspheres was almost the same as the experimental results. Other release rates can be obtained by changing the gelatin concentration, as a result, the pre-programmed release rate can be easily manipulated, and the large initial burst release inhibited.

Gelatin post-coating could improve initial burst loss greatly. This is probably because hydrogen bonding between the PLA/PEG copolymer and the gelatin amine group makes the microspheres encapsulation of the drug more stable.

\section{Conclusions}

Introducing polyethylene glycol into polylactic acid homopolymers could decrease the acidity of degraded products, increase the degradation rate, and increase the release rate of drugs, as well as producing higher burst release. Gelatin coating by dipping microspheres into a gelatin solution greatly reduces the burst effect. The release rate was inversely proportional to the concentration of gelatin being coated. The gelatin-coated microspheres not only reduced the initial burst, but also provided well-controlled drug release. While the possibility of interference with the characteristic of long blood-circulating time by the gelatin post-coating needs further study.

\section{Acknowledgements}

This research was supported by the National Science Council, and Chinese Petro. Corp., Taipei, Taiwan NSC88-CPC-E-002-026.

\section{References}

Cohn, D., Younes, H., 1988. Biodegradable PEO/PLA block copolymers. J. Biomed. Mater. Res. 22, 993-1009.

Flory, P.J., 1967. Principles of Polymer Chemistry, 6th Ed. New York, p. 605.

Gogolewski, S., Jovanovic, M., Perren, S.M., Dillon, J.G., Hughes, M.K., 1993. Tissue response and in vivo degradation of selected polyhydroxyacids: polylactides (PLA), poly(3-hydroxybutyrate) (PHB), and poly(3-hydroxybutyrate-co-3-hydroxyvalerate) (PHB/VA). J. Biomed. Mater. Res. 27, 1135-1148.

Gref, R., Minamitake, Y., Peracchia, M.T., et al., 1994. Biodegradable long-circulating polymeric nanospheres. Science 263, 1600-1603.

Gupta, R.K., Alroy, J., Alonso, M.J., Langer, R., Siber, G.R., 1997. Chronic local tissue reactions, long-term immunogenicity and immunologic priming of mice and guinea pigs to tetanus toxoid encapsulated in biodegradable polymer microspheres composed of poly lactide-co-glycolide polymers. Vaccine 15, 1716-1723.

Hrkach, J.S., Peracchia, M.T., Domb, A., Lotan, N., Langer, R., 1997. Nanotechnology for biomaterials engineering: structural characterization of amphiphilic polymeric nanoparticles by ${ }^{1} \mathrm{H}-\mathrm{NMR}$ spectroscopy. Biomaterials 18 , 27-30.

Huang, Y.Y., Chung, T.W., Tzeng, T.W., 1997. Drug release from PLA/PEG microparticulates. Int. J. Pharm. 156, 915.

Illum, L., Jacobsen, L.O., Muller, R.H., Mak, E., Davis, S.S., 1987. Surface characteristics and the interaction of colloidal particles with mouse peritoneal macrophages. Biomaterials 8, 113-117.

Miyamoto, S., Takaoka, K., Okada, T., Yoshikawa, H., Hashimoto, J., Suzuki, S., Ono, K., 1992. Evaluation of polylactic acid homopolymers as carriers for bone morphogenetic protein. Clin. Orthop. 278, 274-282.

Miyamoto, S., Takaoka, K., Okada, T., et al., 1993. Polylactic acid-polyethylene glycerol block copolymer-A new biodegradable synthetic carrier for bone morphogenetic protein. Clin. Orthop. Relat. Res. 294, 333-343.

Patel, N., Padera, R., Sander, G.W., et al., 1998. Spatially controlled tissue engineering on biodegradable polymer surface. Proceed. Intern. Symp. Control. Rel. Bioact. Mater. 25, 109-110.

Po, A.L., Mhando, J.R., 1984. Formulation of sustained-release products: dissolution and diffusion-controlled release from gelatin films. Int. J. Pharm. 20, 87-98. 
Mann, S., Ozin, G.A., 1996. Nature 382, 313.

Sampath, S.S., Garvin, K., Robinson, D.H., 1992. Preparation and characterization of biodegradable poly (L-lactic acid) gentamicin delivery systems. Int. J. Pharm. 78, 165174.

Singh, P.C., Stockar, U., 1996. A simple penetration model for mass transfer in packed beds and strings of spheres. Chem. Eng. Proc. 35, 343-347.

Zhu, K.J., Lin, X., Yang, S., 1990. Preparation, characterization and properties of polylactide(PLA)-poly(ethyleneglycol) (PEG) copolymers: a potential drug carrier. J. Appli. Polymer Sci. 39, 1-9. 